\title{
Long-term evolution of Earth's continental surface elevation
}

\author{
Shanan E. Peters ${ }^{1}$ and David B. Rowley ${ }^{2}$ \\ 1. Dept. Geoscience, University of Wisconsin-Madison, Madison WI <peters@geology.wisc.edu> \\ 2. Dept. of the Geophysical Sciences, University of Chicago, Chicago IL < rowley@geosci.uchicago.edu $>$
}
Please note that despite having undergone peer-review, this manuscript has yet to be formally accepted for publication. Subsequent versions of this manuscript may have slightly different content. If accepted, the final version of this manuscript will be available at the 'Peer-reviewed Publication DOl' link on the right-hand side of this webpage. Please feel free to contact any of the authors; we welcome feedback.




\title{
Long-term evolution of Earth's continental surface elevation
}

\author{
Shanan E. Peters ${ }^{1, a)}$ and David B. Rowley ${ }^{2}$ \\ ${ }^{1)}$ Department of Geoscience, University of Wisconsin-Madison, Madison, WI 53706, \\ USA. \\ ${ }^{2)}$ Department of the Geophysical Sciences, University of Chicago, Chicago, IL 60637, \\ USA.
}

(Dated: 15 March 2021)

\begin{abstract}
Determining the timescale over which continental surface elevation (hypsometry) evolves is difficult because it reflects a combination of isostasy and dynamic topography operating in concert with erosion and deposition. Here, we use 252 million year old and younger shallow marine sediments exposed at the surface as tracers of net change in continental surface elevation relative to the geoid surface defined as sea level between two points in time: today and when those sediments were deposited. In aggregate, we find that the elevations of Triassic and younger surface-exposed shallow marine sediments closely mirror global continental hypsometry. However, dispersion in the elevations of marine sediments increases with increasing depositional age away from a constant modal elevation of $\sim 0 \mathrm{~m}$. This empirical age-elevation relationship is consistent with the expectations of a diffusion model, wherein shallow marine sediments are continually deposited near $0 \mathrm{~m}$ in the submerged and initially subsiding regions of the continents and then undergo vertical displacements down and up with a constant stochastic distribution of rates. When such a model is tuned to empirical age-elevation data, an asymptotically-stable distribution of surface elevations congruent with observed continental hypsometry emerges on a timescale of $10^{7}-10^{8}$ years.
\end{abstract}

Keywords: continental hypsometry; surface elevation; marine sediments; surface uplift; sea level

\section{INTRODUCTION}

Isostasy and dynamic topography result in crustal surface uplift or subsidence and their time-integrated effects acting in concert with erosion and deposition determine global hypsometry ${ }^{1,2}$. Isostasy primarily reflects the correlation of surface elevation with crustal thickness, with secondary contributions from variations in density and/or flexure. Dynamic topography, that part of Earth's topography that is not accounted for by isostasy, results from stresses acting on the lithosphere that are associated with radial components of mantle flow as well as thermal and chemical buoyancy residing within the mantle lithosphere ${ }^{2}$. The timescales of change in continental hypsometry, then, depend on the rates of change of crustal thickness, with contributions from erosion, deposition and dynamic topography. Thermochronology and cosmogenic estimates of local erosion capture aspects of denudation ${ }^{3}$, but these do not provide estimates of changes in surface elevation. In principle, paleoaltimetry can yield estimates of surface uplift ${ }^{4}$, but most approaches are capable of detecting only large-magnitude uplifts associated with mountain building. It is, therefore, difficult to assess how and over what timescale Earth's continental surface elevation evolves ${ }^{1,5,6}$.

The present-day elevations of globally-distributed shallow marine sediments (Fig. 1) are useful tracers of net vertical continental surface motion because geological observations constrain their initial elevation to within a few tens of meters of sea level ${ }^{7}$ and because determining their present-day elevation is straight-forward. Thus,

a) Electronic mail: peters@geology.wisc.edu regardless of the specific history of burial, uplift, and exhumation/erosion after deposition, the present-day elevation of surface-exposed shallow marine sediments unambiguously constrain the elevation of Earth's surface relative to sea level at two points in time: today and when those sediments were deposited. A global scale analysis is advantageous because the integrated vertical motions of continental lithosphere are likely to be approximately zero-centered on long timescales, whereas isolated regions are free to undergo directional shifts in elevation due to changes in local boundary conditions.

\section{DATA}

We use data from the Paleogeographic Atlas Project (PGAP) at the University of Chicago ${ }^{8}$ and elevation data from the ETOPO1 global digital elevation model ${ }^{9}$. The PGAP data represent a globally comprehensive survey of surface-exposed rock units grouped into one of 15 chronostratigraphic stages (average duration $5 \mathrm{Myr}$ ) from the Early Triassic Induan stage (252 Ma) to the recent; ages are based on the updated international chronostratigraphic timescale ${ }^{10}$. Each PGAP location consists of latitude and longitude coordinates and descriptions of sedimentary deposits and their environments of deposition. Here, we use 11,251 locations (Fig. 1) that preserve marginal marine and marine shelf sediments estimated to have been deposited in water depths of less than $\sim 50 \mathrm{~m}^{7}$. Because the geographic coordinates of each PGAP location are resolved to the nearest tenth of a degree, the elevation of each location was estimated using the minimum, maximum, and mean elevation from the 36 surrounding one arc-minute ETOPO1 grid cells. The global and statistical nature of our analysis, combined 


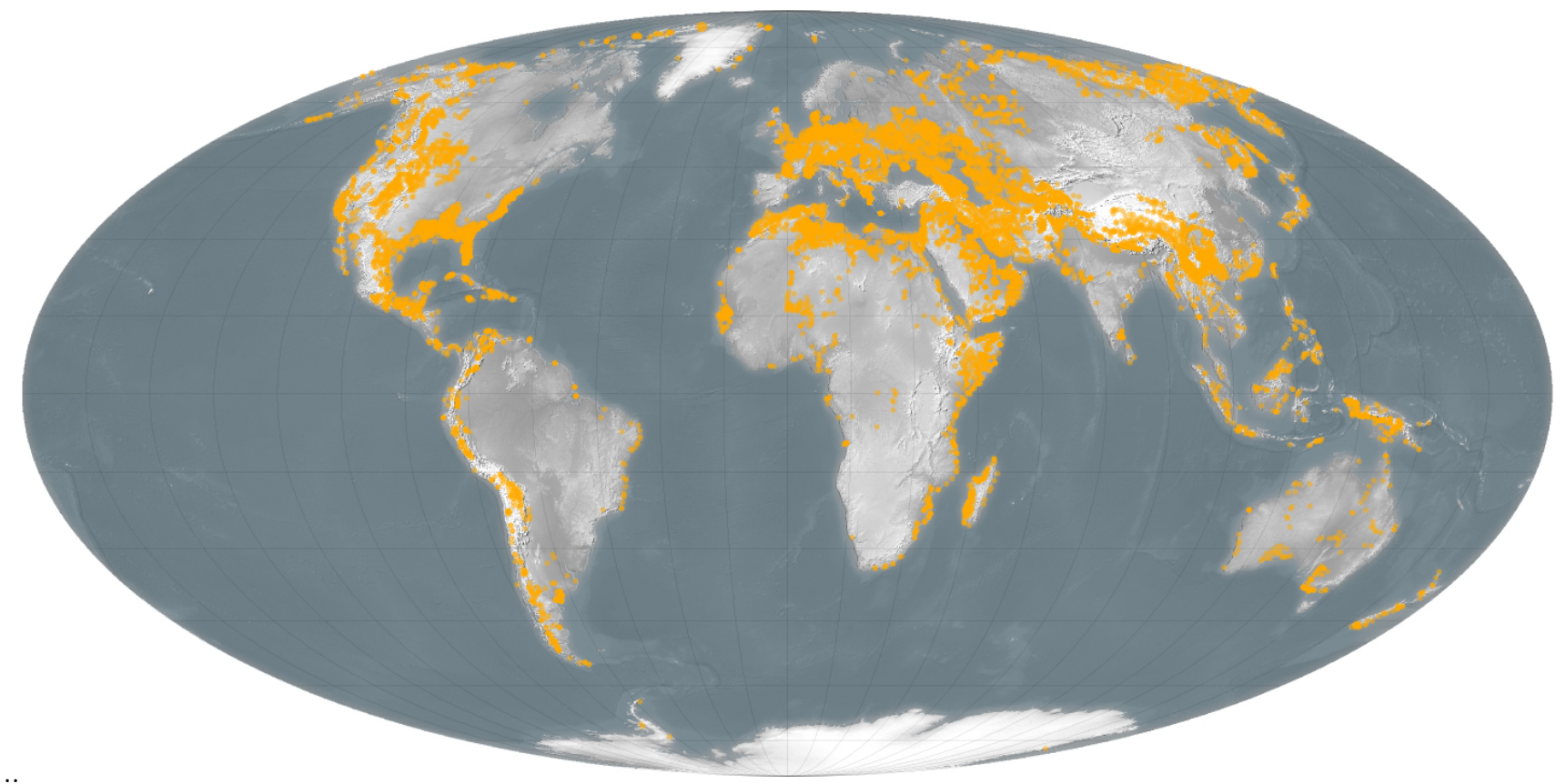

FIG. 1. All 11,251 shallow marine sediment locations from the PGAP compilation plotted on shaded relief ETOPO-1 DEM.

with the inherent crustal length-scale of surface uplift, renders random error in our estimated elevation of each observation of little importance. Results are not sensitive to which ETOPO1 elevation estimate is used (Fig. 6 ) and they are robust to data culling and subsampling (Fig. 7).

\section{RESULTS AND MODEL DESCRIPTION}

In aggregate, the surface elevations of exposed Triassic and younger shallow marine sedimentary rocks closely mirror global present-day continental hypsometry (Fig. 2 ), with a combined mean elevation of $711 \mathrm{~m}$. This falls between the approximately $793 \mathrm{~m}$ mean elevation from ETOPO1-Ice regridded at a tenth of a degree resolution and the $649 \mathrm{~m}$ mean elevation from ETOPO1-Bed regridded at a tenth of a degree. It is also close to the 797 $\mathrm{m}$ mean elevation of the continents estimated from other surface elevation data ${ }^{11}$.

The fact that Triassic and younger shallow marine sediments exposed at the surface are widely distributed with respect to present-day surface elevation and, in aggregate, closely reproduce present-day global continental hypsometry is a fundamental observation that relates directly to the processes governing vertical surface uplift and subsidence. Specifically, it raises the possibility that the timescale required to modify an initially low elevation surface (defined by the formerly ocean covered portions of the continents) and to converge on present-day global continental hypsometry is captured by the elevation-age relationships of shallow marine sediments.

When the present-day elevations of surface-exposed marine sedimentary rocks are segregated into groups based on their geological age of deposition (Fig. 3), there is a significant and systematic change in the distribution of elevation as a function of sediment depositional age. Notably, there is a significant increase in mean elevation with increasing age, from $0 \mathrm{~m}$ in young marine sediments to over $1,000 \mathrm{~m}$ among Triassic shallow marine deposits (Fig. 4a). The general pattern of increasing mean elevation with increasing age persists regardless of which ETOPO1 elevation estimate is used (Fig. 6), after random culling of data (Fig. 7), and after removing all samples located within active orogenic zones (Fig. 8).

The observed increase in mean elevation as a function of geological age is closely mirrored by an increase in the variance in elevations (Fig. 4B). Indeed, the observed increase in mean elevation with increasing age is not driven by a change in modal elevation. Instead, the increase in mean reflects a systematic increase in the dispersion of elevations away from a constant mode of $\sim 0 \mathrm{~m}$ (Fig. 3).

Increase in the variance of tracer positions as a function of time with no change in mode is characteristic of a diffusive process:

$$
V(t)=C_{\theta} t^{\theta}
$$

where $V(t)$ is the mean square displacement (variance) at time $t$ and $C_{\theta}$ is the normal coefficient of diffusion for the case $\theta=1$. That is, if all tracer points are independent, there is no spatial or temporal correlation in their motions, and the system is unbounded (i.e., all positions, regardless of magnitude, are possible), then normal diffusion applies. In such cases, the distribution of point positions is Gaussian around the starting position, with variance in position increasing as a linear function of time (i.e., $\theta=1$ ). If any one or more of these conditions are violated, then $\theta \neq 1$ and the system will exhibit anomalous diffusion ${ }^{12,13}$. When $\theta<1$ the system is subdiffusive and when $\theta>1$ the system exhibits superdiffusive behavior.

Linear regressions of the observed variance in eleva- 


\begin{tabular}{|c|c|c|c|c|c|c|}
\hline ETOPO1 Estimate & Model & $\theta$ & $C_{\theta}$ & $r^{2}$ & P-value & $C_{\theta_{\text {cor }}}$ \\
\hline average & normal & 1.00 & $5,918 \mathrm{~m}^{2} / M y r$ & 0.81 & $8 x 10^{-6}$ & $17,037 \mathrm{~m}^{2} / M y r$ \\
\hline average & anomalous & 0.68 & $38,095 \mathrm{~m}^{2} / M^{\prime} r^{\theta}$ & 0.86 & $1 x 10^{-6}$ & - \\
\hline maximum & normal & 1.00 & $6,772 \mathrm{~m}^{2} / M y r$ & 0.84 & $3 x 10^{-6}$ & $20,004 \mathrm{~m}^{2} / M y r$ \\
\hline maximum & anomalous & 0.57 & $81,739 \mathrm{~m}^{2} / M^{\prime} r^{\theta}$ & 0.86 & $1 x 10^{-6}$ & - \\
\hline minimum & normal & 1.00 & $5,171 \mathrm{~m}^{2} / M y r$ & 0.80 & $4 x 10^{-6}$ & $14,230 \mathrm{~m}^{2} / \mathrm{Myr}$ \\
\hline minimum & anomalous & 0.75 & $21,253 \mathrm{~m}^{2} / \mathrm{Myr}^{\theta}$ & 0.85 & $2 x 10^{-6}$ & - \\
\hline
\end{tabular}

TABLE I. Description of linear and power-law fits to the observed elevation variance-age data following Equation 1. In these fits, the intercept was not forced to zero because the distribution of elevations of Pleistocene marine sediments (at $\sim 0 \mathrm{Ma}$ ) have non-zero variance due to a combination of error in the estimate and uplift over short amounts of time (see Fig. 3 ). $C_{\theta_{\text {cor }}}$ is the half-normal distribution corrected estimate for the $C_{\theta}$.
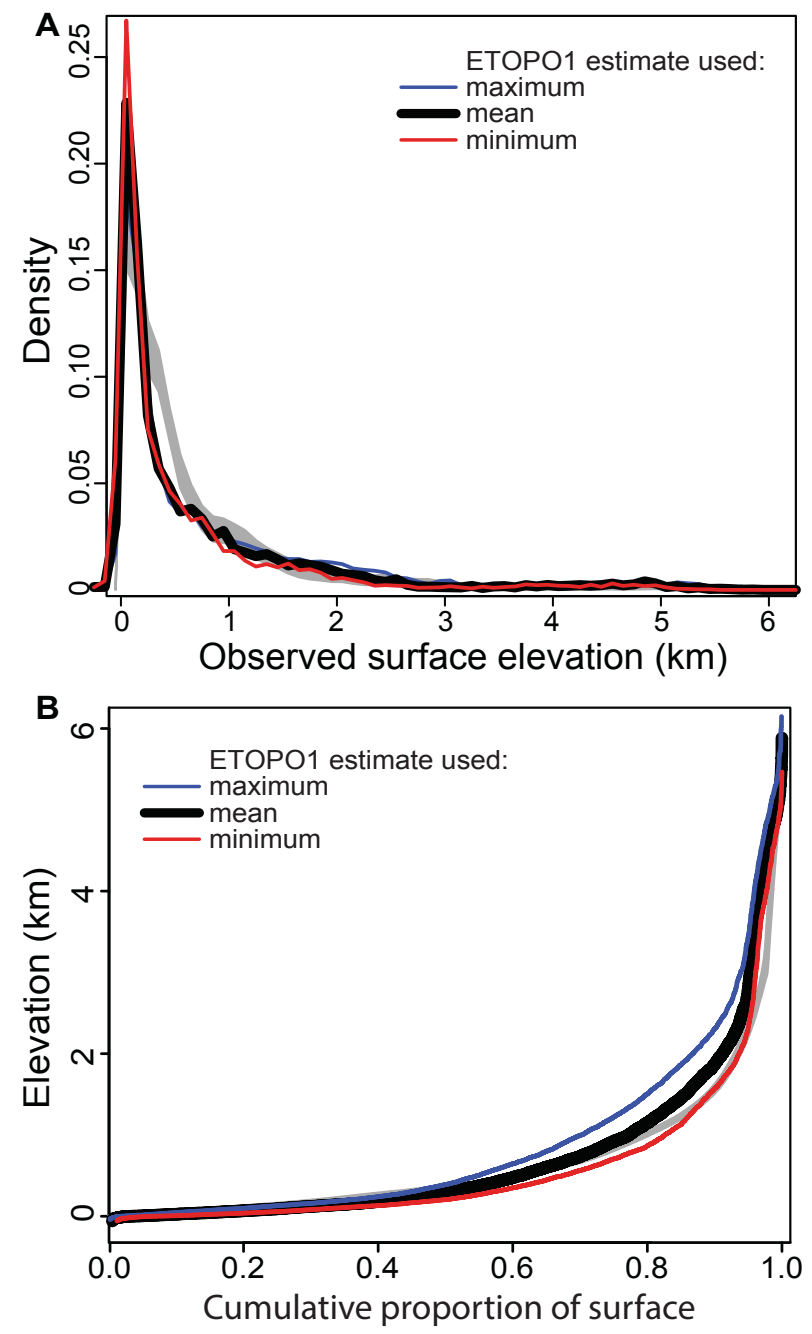

FIG. 2. Global continental hypsometry and marine sediment elevations. A. Density distributions of elevations. B. Same data, but on cumulative $\mathrm{x}$-axis and elevation vertical axis, the familiar representation of continental hypsometry. Observed global continental hypsometry is in gray, with error bounds $( \pm$ 2 standard deviations) generated from 1,000 replicate samples of 11,251 randomly-drawn coordinates from ETOPO1. Observed marine sediment elevations, generated using different conventions for assigning point locations with relatively low precision an elevation from the ETOPO1 grid cells. Elevations estimated using different values from the ETOPO1 grid intersecting and surrounding each location are shown in blue, black and red lines. tion vs. geologic age provide fits with adjusted $r^{2} \geq 0.8$ (see Table 1 for all regression results). Power law fits to the elevation-age data provide better overall descriptions, with $r^{2} \geq 0.85$ and more normally-distributed residuals. In all cases, $\theta<1$, which is indicative of subdiffusive behavior. Subdiffusion occurs in random walk models when the distribution of waiting times between steps has high variance. In other words, when there are "sticking points" that can trap tracers in regions of comparative stability for extended periods of time before undergoing comparatively large and abrupt displacements. Such a scenario is a very good description of large areas of initially low-elevation continental crust flooded by shallow seas. Much of this area could remain rather stable for long periods of time, undergoing slow isostatic and dynamic topographic adjustments before being caught up in plate boundary interactions. For the purpose of model data comparison, it is important to note that subdiffusion results in highly kurtotic, non-normal distributions of tracer positions, which is consistent with the observed non-normality of marine sediment elevations (Fig. 3; Shapiro tests for normality of all reflected distributions, $\left.P<10^{-16}\right)$.

The advantage of any diffusive model is that the aggregate behavior of a closed system can be characterized, as a whole, using empirically grounded observations of the positions of particles (i.e., geographic locations with shallow marine sediment at the surface). Several diffusion models yield reasonably good fits to the observed age-elevation distributions for these shallow marine sediments. One such model ${ }^{14}$ describes the waiting times between successive displacements as:

$$
w(\tau)=\frac{\alpha / \tau_{0}}{\left(1+\tau / \tau_{0}\right)^{1+\alpha}}
$$

where $w(\tau)$ is the waiting time probability density, $\tau_{0}$ is unit time, and $0<\alpha<1$. Step sizes at $w(\tau)$ were drawn from a normal distribution with a mean of 0 and a standard deviation of $w_{s}$. In the example parameterization presented here, $\alpha=0.23, w_{s}=950 \mathrm{~m}$, and an upper boundary was imposed at $+9,000 \mathrm{~m}$, past which points underwent attrition (the probability of achieving such high elevations is low and therefore the effect is negligible; see Fig. 4C). The starting elevations of each point were randomly drawn from a uniform distribution between -150 and $150 \mathrm{~m}$; all points were also incremented in each time step by drawing a random deviate from a 

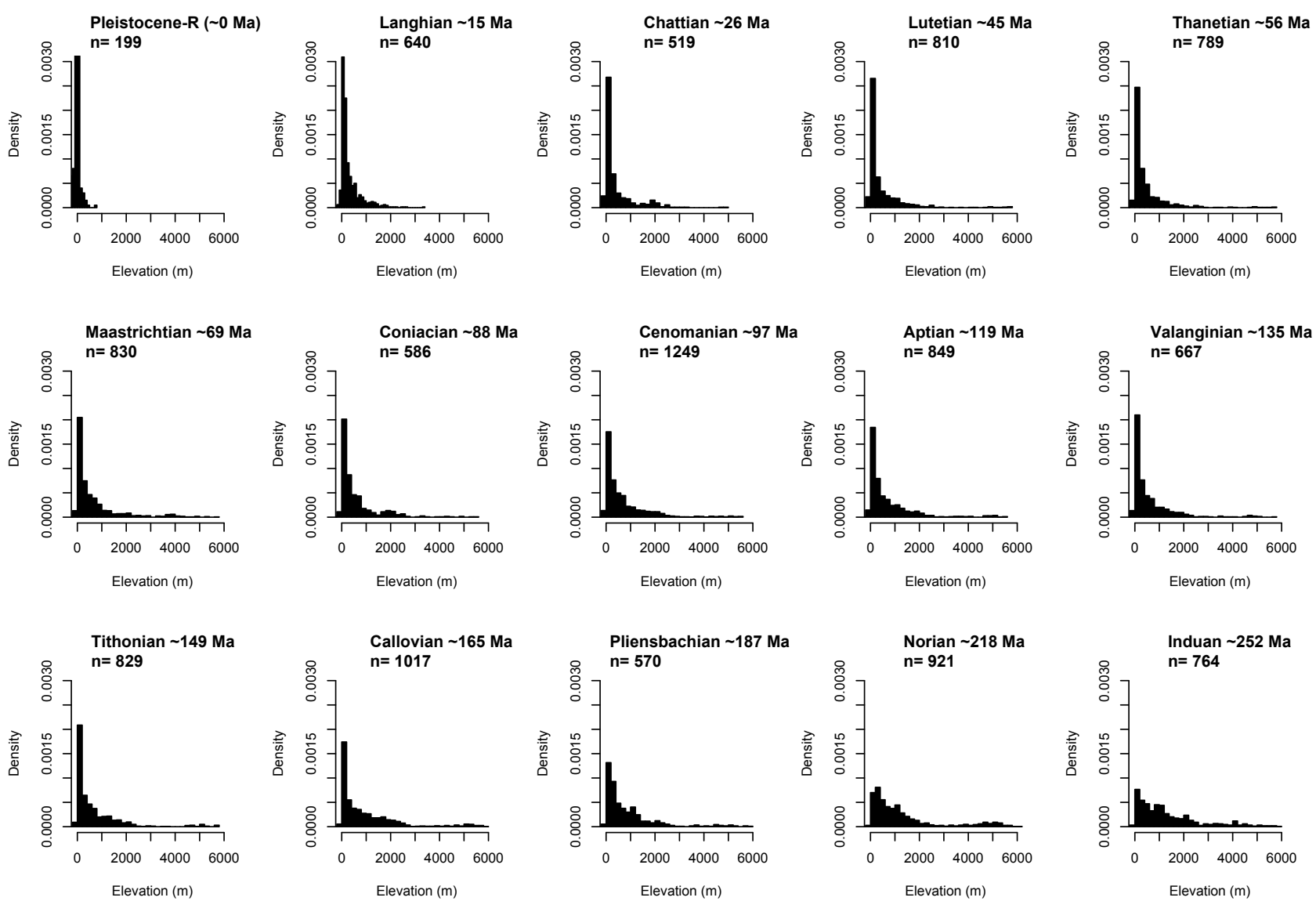

FIG. 3. Empirical density distributions of elevations of surface-exposed shallow marine sediment. Elevations in each temporal cohort are based on average ETOPO1 elevation estimate. Number of locations used to generate each distribution (n) also shown. All distributions are plotted on identical axes. Shapiro tests of normality for distributions reflected by randomly selecting $50 \%$ of the data and multiplying by -1 all have $P \leq 10^{-16}$, indicating non-normality. Note that the modal elevation for all cohorts remains at or near $0 \mathrm{~m}$. Change in elevation distributions is systematic with increasing age and driven by an increase in the dispersion of elevations away from a constant modal elevation of $\sim 0 \mathrm{~m}$.

normal distribution with a mean of $0 \mathrm{~m}$ and a standard deviation of $15 \mathrm{~m}$. Note that these two components of the model fully encompass the proposed magnitude of Triassic-Recent eustatic sea level variation ${ }^{15,16}$ as well as variation in the initial water depths in which the sediments were deposited. Model predictions (Fig. 4) are broadly similar under a range of values for $\alpha, w_{s}$, the range of starting positions, and the standard deviation of the random normal deviate.

It is important to note here that our emphasis is not on the value of any one model or the specifics of modeldata fit or regression coefficients (Table 1). Instead, we focus on the general behavior and explanatory power of such a model in describing the age-elevation data and the implied timescale required to generate continental hypsometry.

\section{DISCUSSION}

An empirically-tuned diffusion model predicts many aspects of the global age-elevation frequency distributions of shallow marine sediments (Fig. 4). Triassic sediments, which are higher in observed mean and variance than predicted, are the largest departures. These positive residuals can be attributed to a disproportionately large number of Triassic sample locations on the Tibetan Plateau, which results in a small mode at high elevation (Fig. 3). Imposing temporal variability in the starting elevation of marine sediments to reflect putative eustatic sea level changes over geologic time, even with an amplitude of 300 meters $^{15,16}$, has no effect on model predictions after only a few million years because vertical motion is cumulative and large in magnitude by comparison (Fig. 3, 4a). There is, therefore, little signature of eustatic sea level remaining in the present-day elevations of shallow marine sediments after they have aged only few million years. This result is consistent with the finding that tectonic and dynamic topography controls the long-term history of continental marine inundation ${ }^{17,18}$ and with the suggestion that there has been only $25 \pm$ $22 \mathrm{~m}$ of eustatic sea level change from the Jurassic to Eocene relative to continental ice-free and glacial isostatically corrected crustal hypsometry ${ }^{19}$.

When the diffusion model is run for $252 \mathrm{Myr}$ and all elevations $\geq 0 \mathrm{~m}$ are included in a single frequency dis- 

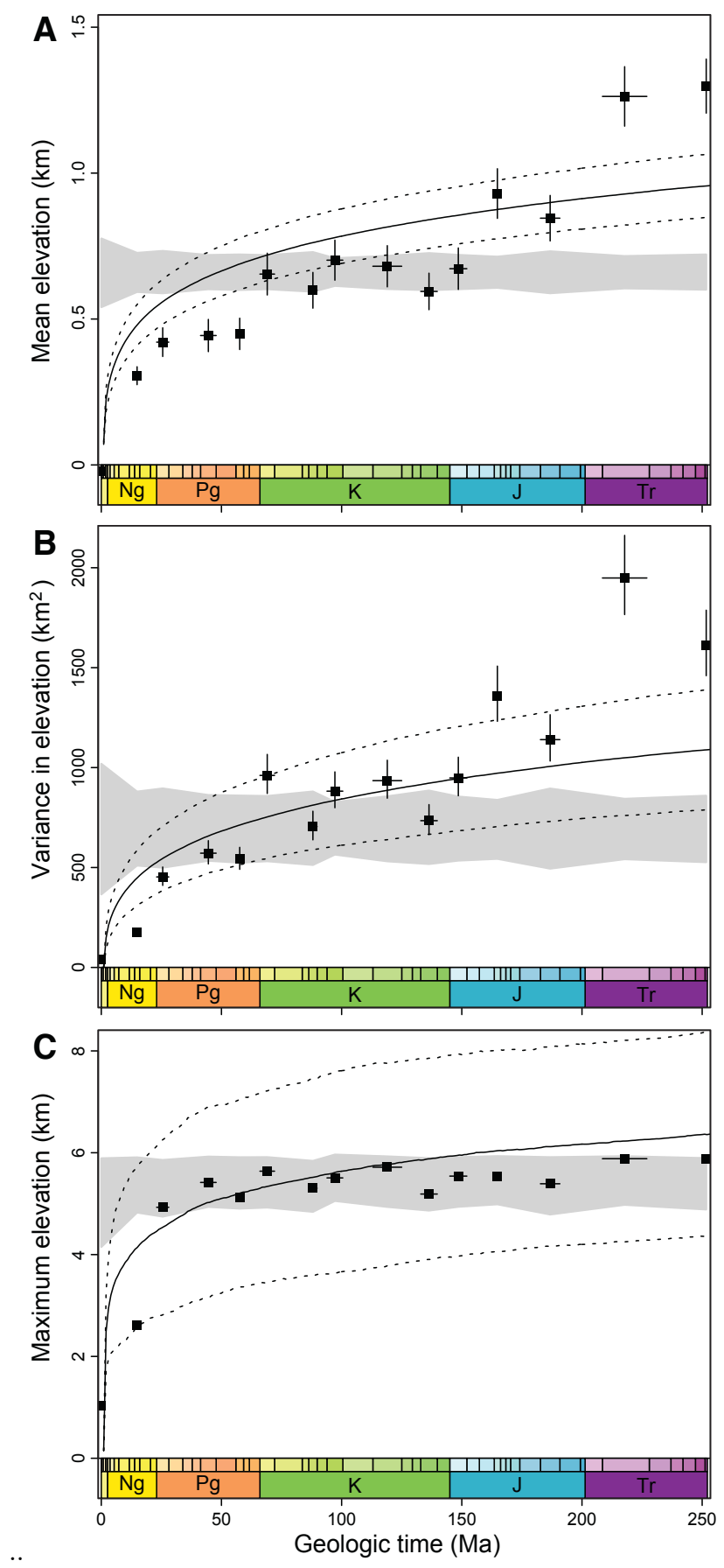

FIG. 4. Observed and model-predicted elevations of marine sediment vs. geologic age. Observations shown as points; $\mathrm{x}-$ error bars correspond to the duration of time bin that marine sediments were assigned to in the PGAP compilation and yerror bars show \pm 2 standard errors of the estimates in that bin (see Fig. 3 for raw empirical distributions summarized here by statistical moments). Gray fields show expected value based on a random sample of ETOPO1 with a sample size equal to that available for each time bin. Dotted lines are two standard deviations about the expected value (solid line) of 1,000 iterations of a diffusion model run continuously over the timescale shown. Each iteration of this model had 750 points, comparable to the average number of empirical observations in each time bin (black points, see also Fig. 3). A: Mean elevation. B: Variance in elevation. C: Maximum elevation.

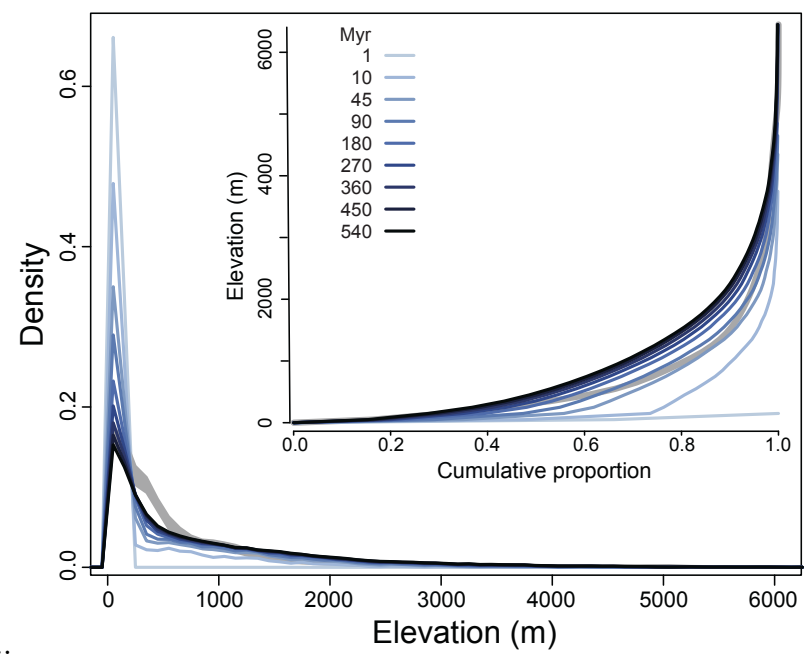

FIG. 5. Model-predicted distribution of surface elevations and observed continental hyposometry. Gray heavy lines (mostly obscured by blue model predictions) show hypsometry derived from randomly sampling ETOPO1. Range of gray bar shows the mean and two standard deviations of 1,000 bootstrap replicate samples of 11,251 randomly drawn ETOPO1 surface elevations (as in Fig. 2). Blue lines show hypsometry predicted by running the model shown in Fig. 4 for 541 Myr. Inset shows same data as main figure, but with elevation plotted against cumulative proportion of data.

tribution, irrespective of age, hypsometry similar to that observed for the continents today emerges (Fig. 5) with a mean elevation of $\sim 800 \mathrm{~m}$. When extending the model to encompass the $541 \mathrm{Myr}$ duration of the Phanerozoic, the predicted mean continental surface elevation increases to $\sim 900 \mathrm{~m}$, but does not markedly change the expected hypsometry, which evolves indefinitely but asymptotically (Fig. 5). Thus the $\sim 250 \mathrm{Myr}$ time frame of the data used here are sufficient to capture most of the expected change in the age-elevation relationship (modelled global mean elevation increases by only $0.8 \mathrm{~m} / \mathrm{Myr}$ after $252 \mathrm{Myr}$ and by less than $0.4 \mathrm{~m} / \mathrm{Myr}$ after $541 \mathrm{Myr}$ ). Extrapolation beyond the Phanerozoic is unlikely to be informative due to the large discontinuity in shallow marine sediment abundance that occurs at the start of the Phanerozoic, which is indicative of a major change in the nature of marine sediment accumulation on the continents, a period of Precambrian continental denudation, or both ${ }^{20,21}$.

Long-term modeled rates of vertical surface uplift, measured as changes in elevation from time $t_{i}$ to time $t_{i+1}$, are $\sim 17 \mathrm{~m} / \mathrm{Myr}$ on average. This is comparable to long-term sediment accumulation rates measured over the same unit duration ${ }^{2-24}$. If rates of sedimentation measured on Myr timescales are controlled by rates of crustal subsidence, then agreement in vertical surface displacements measured by sediment thickness-duration and age-elevation relationships is expected and, indeed, this is a fundamental requirement of a diffusion-type model. It is important to note that there is no predicted change in mean elevation $(\sim 0 \mathrm{~m})$ of marine sediment vs. as a function of its depositional age. That is, shallow marine sediments of all ages are expected to remain at $\sim 0 \mathrm{~m}$ in elevation, on average, with only some fraction of those 
sediments travelling downward into the subsurface to get buried by younger materials, and an equal number being uplifted to get exposed and subject to erosion and other surface processes. Thus, the expectation of this basic diffusion model is that there is a distribution of negative elevations for shallow marine sediments in the subsurface that is at comparable to that which is observed at the surface (Fig. 3). The thickness and ages of sediment in between a sedimentary unit in the subsurface and one at the surface will reflect the specific subsidence-uplift and sedimentation/erosion history at each location.

The signature of erosion is not readily apparent in the marine sediment elevation data (Fig. 4) nor is it explicitly included in the diffusion model. However, most shallow marine sediments exposed at the surface today were buried by some depth of overlying sediment before being returned to the surface by exhumation. Erosion, therefore, clearly plays a central role in the history of most marine sediments exposed at the surface today. However, exhumation is distinct from surface uplift ${ }^{1}$ and we are considering the surface elevations of rocks which, by definition, have not been lost to erosion. It is also possible that the signature of erosion would be clearly expressed if we had comprehensive data on the subsurface elevations of shallow marine sediments of the same ages and over a comparable, comprehensive global-scale tabulation of continental crust. Such data might reveal that the distribution of elevations for cohorts of shallow marine sediments in the subsurface are not symmetric with those sediments of the same age and exposed at the surface today (Fig. 3), which is a fundamental requirement of our diffusion model. If erosion were a predominate factor affecting surface-exposed sediments and their elevations, then we might expect to see marine sediments of a given age exhibiting an even greater dispersion of subsurface elevations with increasing age than that which is observed for surface-exposed sediment cohorts (Fig. 3,4).

Although we do not yet have this type of data on the subsurface elevations of shallow marine sediment, we do have data describing the total surviving quantity of shallow marine sediment in the subsurface and surface over this same time interval ${ }^{20,25}$. These data or rock quantity are consistent with some aspects of our results. Specifically, the surviving quantity of shallow marine sedimentary rock, including that in the surface and subsurface, lacks one of the most basic patterns that is expected in all models of sediment cycling and erosion: there is no decline in the quantity of surviving shallow marine sediment with increasing age ${ }^{20,25}$. On the contrary, there is an overall increase in surviving marine sediment quantity with increasing age during most of the Phanerozoic, with variability primarily reflecting changes in how much continental crust is inundated ${ }^{20,25}$. When analyses of marine sediments are spatially integrated over the scale of continents, the quantitative signature of erosion is apparently quite diminished. The possible emergence during the Phanerozoic of an asymptotically-stable continental hypsometry (Fig. 5), driven in part by net growth in the total amount of sedimentary cover on the continents ${ }^{26,27}$, has many implications for the long-term evolution of Earth's surface environment.

\section{ACKNOWLEDGMENTS}

We thank A.M. Ziegler for leading the PGAP initiative and B. Wilkinson and J. Husson for helpful feedback on an early draft of this paper. The manuscript benefited from constructive reviews by D. Sahagian, N. ChristieBlick, B. McElroy, R. Westaway, and P. Rey.

\section{References}

${ }^{1}$ P. England and P. Molnar, Geology 18, 1173 (1990).

${ }^{2}$ A. Forte, W. Peltier, A. Dziewonski, and R. Woodward, Geophysical Research Letters 20, 225 (1993).

${ }^{3}$ P. W. Reiners and M. T. Brandon, Annu. Rev. Earth Planet. Sci. 34, 419 (2006).

${ }^{4}$ D. B. Rowley and C. N. Garzione, Annu. Rev. Earth Planet. Sci. 35, 463 (2007).

${ }^{5}$ P. England and D. McKenzie, Geophysical Journal International 70, 295 (1982).

${ }^{6}$ P. F. Rey and G. Houseman, Geological Society, London, Special Publications 253, 153 (2006).

${ }^{7}$ A. Ziegler, D. B. Rowley, A. L. Lottes, D. L. Sahagian, M. L. Hulver, and T. C. Gierlowski, Annual Review of Earth and Planetary Sciences 13, 385 (1985).

${ }^{8}$ A. Ziegler, G. Eshel, P. M. Rees, T. Rothfus, D. Rowley, and D. Sunderlin, Lethaia 36, 227 (2003).

${ }^{9}$ C. Amante and B. Eakins, National Geophysical Data Center, NOAA 10, V5C8276M (2009).

${ }^{10}$ K. M. Cohen, S. C. Finney, P. L. Gibbard, and J.-X. Fan, Episodes 36, 199 (2013).

${ }^{11}$ D. B. Rowley, The Journal of Geology 121, 445 (2013).

${ }^{12}$ R. Metzler and J. Klafter, Physics reports 339, 1 (2000).

${ }^{13} \mathrm{R}$. Metzler and J. Klafter, Journal of Physics A: Mathematical and General 37, R161 (2004).

${ }^{14}$ T. Neusius, I. M. Sokolov, and J. C. Smith, Physical Review E 80, 011109 (2009).

${ }^{15}$ B. U. Haq, J. Hardenbol, and P. R. Vail, Science 235, 1156 (1987).

${ }^{16}$ K. G. Miller, M. A. Kominz, J. V. Browning, J. D. Wright, G. S. Mountain, M. E. Katz, P. J. Sugarman, B. S. Cramer, N. Christie-Blick, and S. F. Pekar, science 310, 1293 (2005).

${ }^{17}$ M. Gurnis, in Glacial Isostasy, Sea-Level and Mantle Rheology (Springer, 1991) pp. 445-492.

${ }^{18}$ M. Gurnis, Nature 364, 589 (1993).

${ }^{19}$ D. B. Rowley, The Journal of Geology 125, 141 (2017).

${ }^{20}$ S. E. Peters and J. M. Husson, Geology 45, 323 (2017).

${ }^{21}$ C. B. Keller, J. M. Husson, R. N. Mitchell, W. F. Bottke, T. M. Gernon, P. Boehnke, E. A. Bell, N. L. Swanson-Hysell, and S. E. Peters, Proceedings of the National Academy of Sciences 116, 1136 (2019).

${ }^{22}$ P. M. Sadler, The Journal of Geology 89, 569 (1981).

${ }^{23}$ B. H. Wilkinson, Geology 33, 161 (2005).

${ }^{24}$ S. E. Peters, The Journal of Geology 114, 391 (2006).

${ }^{25}$ A. Ronov, V. Khain, A. Balukhovsky, and K. Seslavinsky, Sedimentary Geology 25, 311 (1980).

${ }^{26} \mathrm{~J}$. M. Husson and S. E. Peters, Emerging topics in life sciences 2, 125 (2018).

${ }^{27}$ J. M. Husson and S. E. Peters, Earth and Planetary Science Letters 460, 68 (2017). 

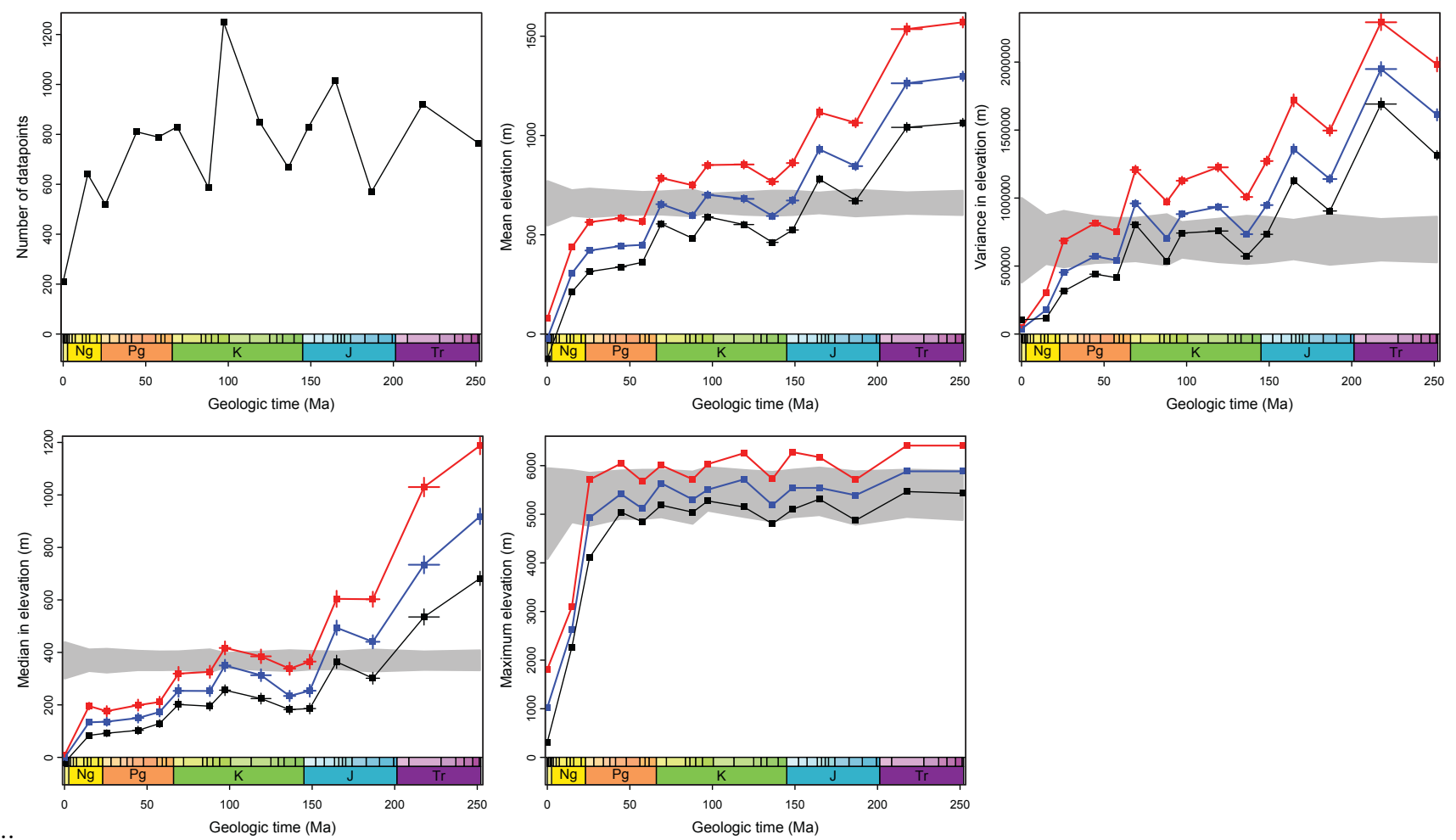

FIG. 6. All statistical moments for the ETOPO1 estimates of surface elevation of marine sediment cohorts (as in Fig. 4). Red, maximum ETOPO1 elevation in each location grid cells. Blue, average elevation. Black, minimum elevation in each location grid cells. Gray areas show mean \pm 2 standard deviations for each statistical moment based on a random sampling of the global ETOPO1 data using the empirical sample size, shown in the panel labeled "Number of datapoints" (upper left).
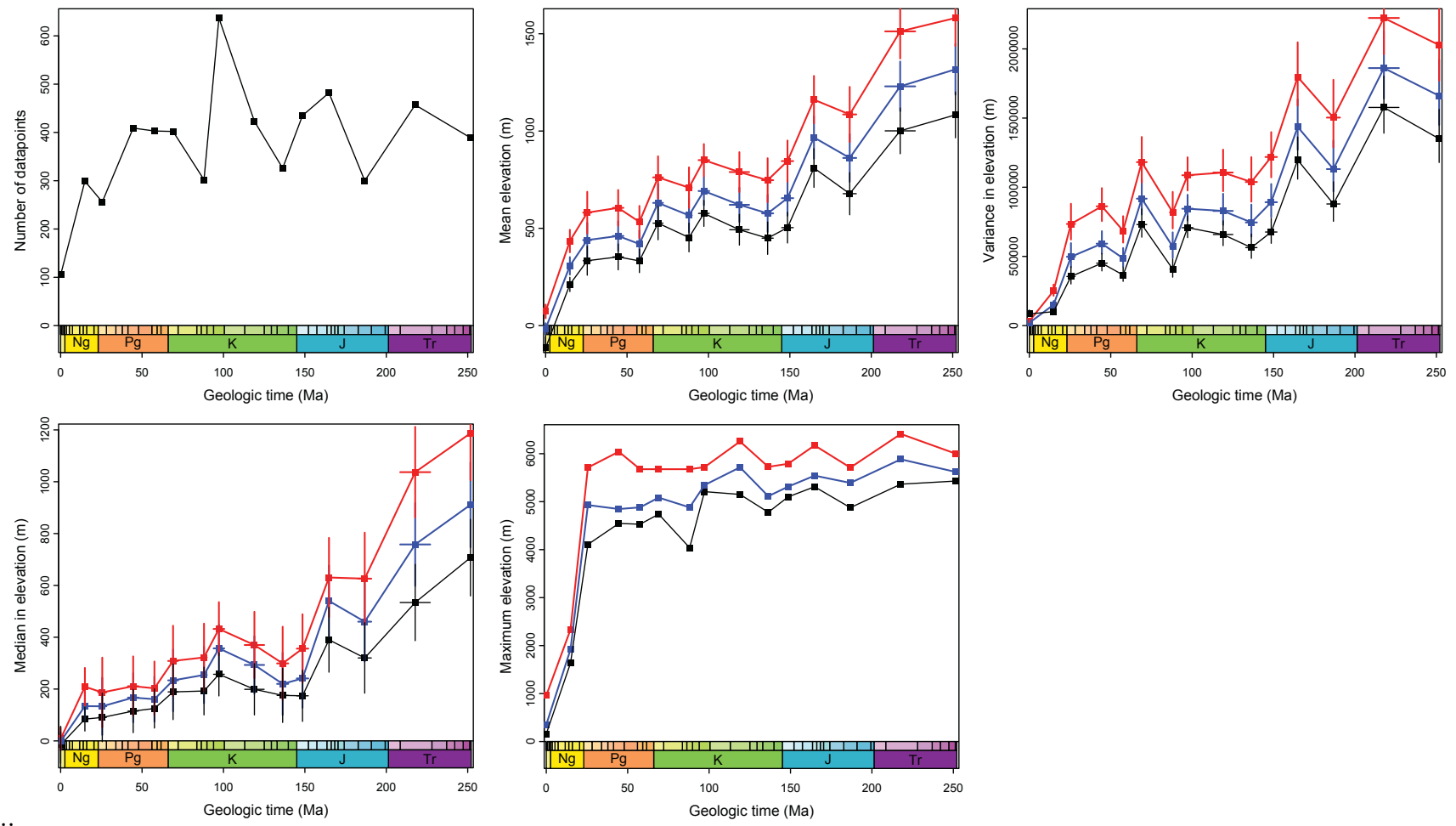

FIG. 7. Statistical moments for all ETOPO1 estimates computed after randomly culling $50 \%$ the empirical data. All symbols as in Fig. 6. Compare to Fig. 4,6. Results are robust to random culling of locations used to estimate surface elevation. 

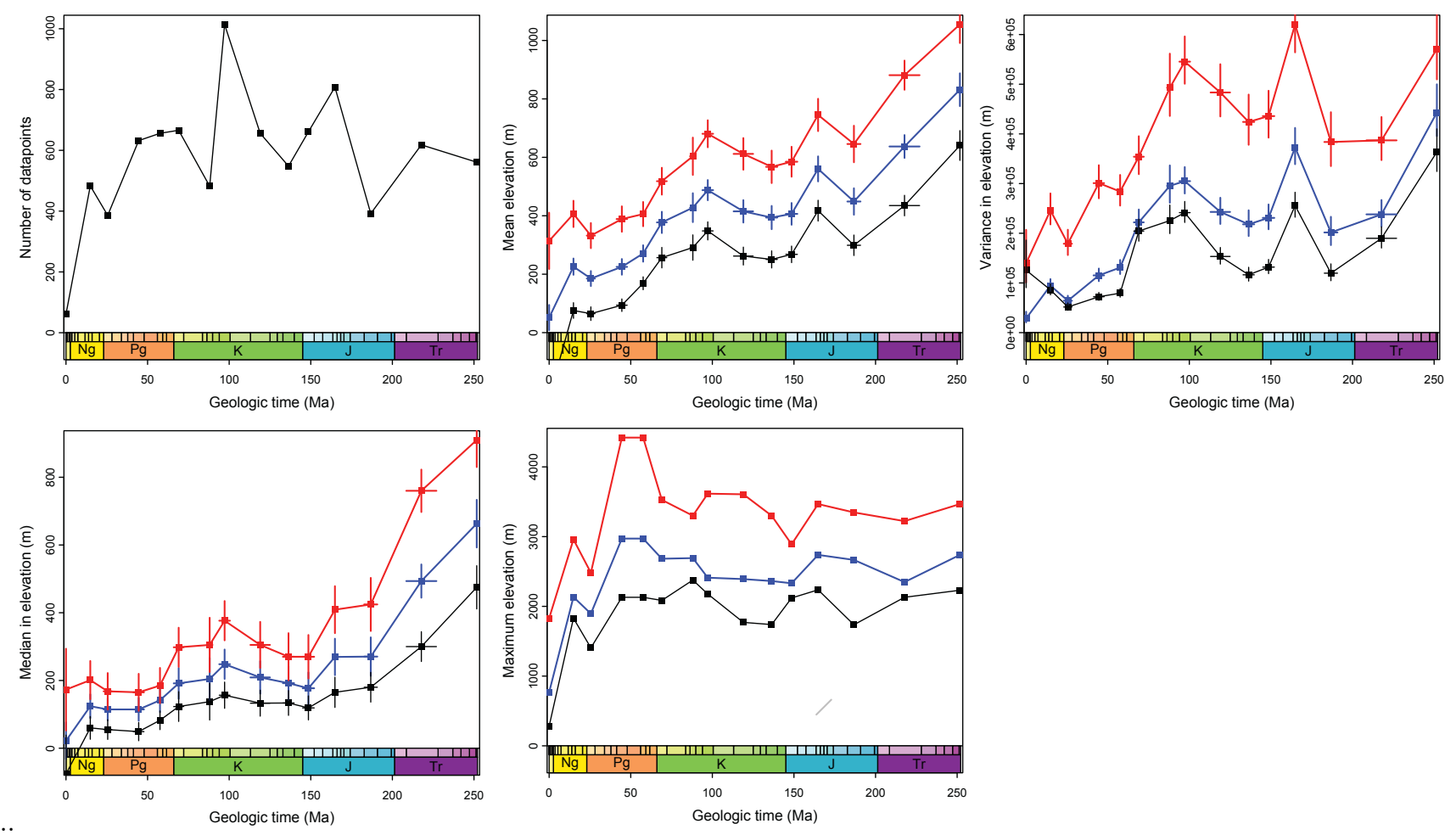

FIG. 8. Statistical moments for all ETOPO estimates, excluding all points in modern orogenic zones. Coloring and labeling of points and lines as in Fig. 6. Points falling within modern orogenic zones were identified semi-quantitatively by using general outlines for modern active orogens and then identifying all empirical shallow marine sediment locations falling within those outlines. These points were then culled from the analysis. A total of 8,624 points remained in the dataset following this cull (a loss of $23 \%$ of the data). Compare to Fig. 4 and Fig. 6. 